\section{A Ligand Field Investigation of the Zirconium-Oxygen System*}

STIG FLODMARK and BJORN ROOS

Institute of Theoretical Physics, University of
Stockholm, Stockholm, Sweden

E xperiments have shown that oxygen is easily solved into zirconium metal. For contents of less than 0.33 oxygen atoms per zirconium atom the oxygen is randomly distributed over the major interstices in the hexagonal close-packed lattice, the structure of which has been determined by X-ray experiments. ${ }^{1}$ These experiments also give the lattice parameters as functions of the oxygen content. On account of the high electronegativity of oxygen it is very probable that the crystal is ionic to some extent. Experimentally this is also known to be true ${ }^{2}$ although the degree of ionization is not known from experiments.

A theoretical investigation of the above properties of the zirconium-oxygen system has now been performed. A ligand-field model, where the overlap between the valence electrons of two different zirconium atoms is neglected, and the oxygen atoms are treated as point charges, was used.

An expression for the cohesive energy of the lattice of the following form is derived:

$$
\varepsilon=\varepsilon_{Z_{\mathrm{r}}}+\varepsilon_{\mathrm{O}}-1 / 2 \varepsilon_{12}+\varepsilon_{\mathrm{M}}
$$

$\varepsilon \mathrm{Zr}_{\mathrm{r}}$ is the energy of the zirconium ion in the ligand field potential; $\varepsilon_{o}$ the energy of the oxygen ions, which are supposed not to interact with the lattice; $\varepsilon_{12}$ is the repulsion between valence-electron systems for different zirconium atoms and $\varepsilon_{M}$ is the Madelung energy of the crystal. The cohesive energy (1) is thus a function of the oxygen charge or the degree of ionization and also of the lattice parameters. It has been found possible to calculate the degree of ionization as well as the lattice parameters by minimizing the energy with respect to these quantities, which has been done for different oxygen contents. The agreement with experimental results for the lattice parameters is remarkably good. Therefore, one may expect that also the ionic charge, determined as a function of

* This investigation is supported by the U.S. Army under the contract No. DA-91-591. EUC-2270. the oxygen content, is roughly correct. The one-electron orbitals used in the energy calculation have been determined from an effective one-electron Hamiltonian where the perturbation due to the neighbouring ions of a zirconium atom in the crystal is represented by a ligand-field potential calculated under the assumption that these ions can be treated as point charges. Pairing all the neighbouring ions into dipoles, all dipoles having at least one ion inside a sphere of certain radius $R$ are taken into account for the calculation of the matrix elements of the potential. The convergence of these matrix elements with increasing $R$ is acceptable only if $R$ is taken to be at least three cell distances. When only the nearest neighbours are taken into account (corresponding to a radius of one cell distance) very poor results are obtained.

Slater orbitals of type $4 \mathrm{~d}, 4 \mathrm{f}, 5 \mathrm{~s}$, and $5 \mathrm{p}$ have been used in the basis set. The $4 \mathrm{~d}$ and $5 \mathrm{~s}$ orbitals correspond to the ground state configuration for $\mathrm{Zr}$. The $4 \mathrm{f}$ orbitals may be supposed to have a rather high occupation number in the crystal on account of their fitting into the hexagonal symmetry. The results of the calculations show, however, that the f-orbital contribution to the ground-state levels is negligible in this case, which may depend on the neglect of overlap between neighbours.

The one-electron energies calculated by means of this method are in agreement with the result obtained by Altmann in his calculation for pure zirconium, ${ }^{3}$ except for the levels containing $p$ orbitals. This is due to the fact that the overlap between adjacent atomic $p$ orbitals is rather large and also that the basis has not been orthogonalized with respect to the core electrons of the zirconium atom. For these reasons much too low one-electron energies have been obtained for the $\mathrm{p}$ orbitals.

The two lattice parameters $a$ and $c$ and the degree of ionization of the oxygen atoms have been calculated by minimizing the energy (1). The obtained values of $a$ agree within one per cent with the experimental values for different oxygen contents. The $c$ value, however, is $8 \%$ too large. According to the results obtained the oxygen ions have a charge of -1.5 for small oxygen contents. On increasing the oxygen content the charge goes towards the value -2 which was found to be the value for the ideal composition $\mathrm{Zr}_{3} \mathrm{O}$. Actually, however, in this composition the oxygen ions take fixed places in the lattice, thus enlarging the unit cell three times.

Acta Chem. Scand. 17 (1963) No. 3 
The results obtained in this investigation will be used in a calculation of the band structure of the zirconium-oxygen system which is proceeding." Full details of the present investigation will be published by one of us (BR) in Arkiv för Fysik.

1. Holmberg, B. and Dagerhamn, T. Acta Chem. Scand. 15 (1961) 919.

2. Lustmann, B. U.S. Atomic En. Comm. Rep. No. NAPO-RM-17, 318, 319 (July 1950).

3. Altmann, S. L. Proc. Roy. Soc. (London) 244 (1958) 153.

4. Flodmark, S. Techn. Rep. No. 1-6, Institute of Theoretical Physics, University of Stockholm, Stockholm, Sweden $(1960-62)$.

Received March 4, 1963.

\section{Isolation of the Major Ganglioside of Human Spleen}

\section{LARS SVENNERHOLM}

Department of Medical Biochemistry, University of Gothenburg, Gothenburg, Sweden

Tn a study of the ganglioside profile of I human brain, a minor component was observed which constituted less than $1 \%$ of the total ganglioside fraction. The small amount isolated did not allow a further characterization of this ganglioside. It was then observed that the major ganglioside of human spleen had the same $R_{F}$-value in two chloroform-methanol-water solvents and in $70 \%$ aqueous 1 -propanol ${ }^{1}$ as the minor brain component mentioned. Because this spleen ganglioside had considerably higher $R_{F}$-value in all the three solvents than the normal basic brain ganglioside, acyl-sphingosine-N-tetrose-N-acetylneuraminic acid, it was assumed to have a more simple carbohydrate moiety and to be related to a ganglioside isolated by Yamakawa and Suzuki ${ }^{2}$ from equine erythrocytes, termed hematoside, which contained fatty acid-sphingosine-hexosesialic acid in the molar ratio $1: 1: 2: 1$.

Experimental. $1.8 \mathrm{~kg}$ of fresh spleen from 18 old-aged people who had died in cardiovas. cular diseases were homogenized in a Turmix

blender, dehydrated and freed from simple lipids by extraction twice with 10 liters of acetone at $+4^{\circ}$. The remaining lipids were extracted twice with 3 liters of chloroformmethanol (C-M), 2:1, v/v, at room temperature and finally in a Soxhlet hot extractor with C-M 1:2. The combined extracts were evaporated, redissolved in C-M 2: $\mathrm{I}$, and filtered. The extract was evaporated again and hydrolysed for $24 \mathrm{~h}$ at $+37^{\circ}$ in aqueous $0.5 \mathrm{~N} \mathrm{KOH}$. After neutralisation to $\mathrm{pH} 5-6$ the lipids were extracted with 6 volumes C-M 2:1. 90-95\% of the gangliosides were extracted together with the other lipids in the organic solvent phase. The gangliosides remaining in the water phase were isolated by extraction of the dialysed and lyophilized water phase with C-M 2:1. The total lipid extract, combined organic and solvent phases, was chromatographed on four columns of $60 \mathrm{~g}$ silicic acid. ${ }^{3}$ The effluents, collected in $25 \mathrm{ml}$ samples, were tested with thin-layer chromatography and the sphingolipids were combined into six main fractions: ceramide-monohexosides, -dihexosides, -trihexosides, -tetra-saccharides, gangliosides and sphingomyelins ${ }^{4}$. Lipid-bound sialic acid (gangliosides) occurred, except in the ganglioside fraction, also in the other fractions which is evident from Table 1 :

\section{Table 1}

Fraction

Ceramide-dihexosides

1) -trihexosides

1) -tetrasaccharides

Gangliosides

Sphingomyelins

It was found that the major ganglioside of all the fractions had the same $R_{F}$-value in thinlayer chromatography (Fig. 1).

The major ganglioside has only been isolated from the ganglioside fraction. This was far from pure as it contained about equal amounts of ceramide-trihexosides, -tetrasaccharides, and gangliosides. Separation of the gangliosides from the other two glycolipids on columns with diethylaminoethyl (DEAE) cellulose and magnesium trisilicate (Florisil) failed. A very satisfactory separation was achieved on thinlayer plates of silica gel $G$ with the technique recently described. ${ }^{5}$ The recovery, calculated from sialic acid analyses, was about $90 \%$. A chromatographically homogeneous ganglioside was crystallized from methanol. $\mathbf{P} 0$, $\mathrm{N}$ 2.15, hexose 28.1, $\mathrm{N}$-acetylneuraminic acid 